PPPL--2564

DE89 004625

\title{
MAGNETIC FLUCTUATIONS ON TFTR
}

R. L. BOIVIN, C. BUSH, F. DYLLA, E. FREDRICKSON, K.M. MCGUIRE, A.W. MORRIS*, J. SCHIVELL,

B. STRATTON, G. TAYLOR, and J. TIMBERLAKE

PRINCETON PLASMA PHYSICS LABORATORY

PRINCETON UNIVEASITY

PRINCETON, N.J. 08543

*Balliol College, Oxford University, U.K.

ABSTRACT

Data from magnetic pick-up loops (Mirnov coils), located on the wall inside the vacuum vessel of TFTR, are used for studying the edge magnetic fluctuations. Experiments, such as impurity injection, gas puffing, and plasma motion, dramatically affect the fluctuations measured by the coils. A quantitative study of the fluctuation levels during these experiments has been made. Pesults show that there are simple relations between the amount of impurities or gas injected and changes in the fluctuation levels. Spatial locations of the fluctuation modes have also been tentatively identified. Finally, different models were studies in order to explain the behaviour and dependence of the fluctuations on the relevant parameters of the plasma. 


\section{INTRODUCTION}

TFTR is a tokamak with a major radius of $2.55 \mathrm{~m}$, a minor radius of $0.80 \mathrm{~m}$, a toroidal magnetic field up to $5.2 \mathrm{~T}$, an electron temperature up to $8 \mathrm{keV}$, an ion temperature up to $30 \mathrm{keV}$, and an electron density up to $8 \times 10^{13} \mathrm{~cm}^{-3}$ (higher with pellet injection). We used two filling gases, deuterium(D) (in the majority of shots) and helium(He). The plasma current ranged, for our experiments, from 0.6 to 1.8 MA. The magnetic fluctuation amplitude, as measured by the nearest coil to the plasma, is of the order of $10^{-4} \mathrm{~T}$, in the 1 to $100 \mathrm{kHz}$ range. All the experiments studied quantitatively were done on ohmic discharges or before neutral beam injection. This allowed us to identify more easily the sources of fluctuations. However, the same behaviour occurred, at least qualitatively, during neutral beam heating and the general case will be discussed in the last section.

Three different experiments were carried out on TFTR. The first consisted of injecting impurities by the laser blow-off technique. In the second experiment a smail amount of gas was introduced (gas puffing), and the third one corisisted of moving the plasma (free expansion of the plasma). Abrupt changes in the magnetic fluctuation level picked up by the Mirnov coils around the torus were observed upon initiation of the experiments. Effects were studied in detail between the frequencies of 100 and $150 \mathrm{kHz}$, although similar effects were observed over the whole spectrum.

\section{METHOD}

The main array of Mirnov coils is composed of 15 coils distributed poloidally as shown in figure 1 . The nearest coil is typically at a distance of $11 \mathrm{~cm}$ from the plasma edge [1]. A Mirnov coil measures the variations $\left(\dot{\mathrm{B}}_{0}\right)$ of the poloidal magnetic fieid at that point. Since the plasma is not located exactly in the center of the torus, a portion of the radial magnetic fluctuations is picked up, although this is small (typically less than $1 \%$ ) and can be neglected. 
The signal originating from the coils is digitized at two different rates: slow $(10 \mathrm{kH}-\mathrm{z})$ and fast $(50$ to $500 \mathrm{kHz}$ ). The signal from one cail (usually the coil \#1, on the inner midpiane) is also directed to a comb filter which divides the signal in 16 frequency bands and is digitized at $500 \mathrm{~Hz}$. The 16 bands cover the $0.2 \mathrm{kHz}$ to $350 \mathrm{kHz}$ frequency range. Each band obtained, measurgd in volts is then time integrated in order to retrieve the magnetic fluctuations. The time integral is approximated by simply dividing by the mean frequency:

$$
\tilde{B}_{\theta}=\frac{v}{n A f}
$$

where we used $f=125 \mathrm{kHz}$ for the mean frequency of the $100-150 \mathrm{kHz}$ band and where the coil sensitivity $n A_{1}$ is equal to $0.570 \mathrm{~m}^{2}$ or $5.7 \times 10^{-2} \mathrm{Vs} / T$. $\Delta \bar{B}_{\theta}$ is then taken to be the drop in the inagnetic fluctuation level during any event (such as an impurity injection). This value is then normalized by the unperturbed poloidal field evaluated at the coil position:

$$
B_{\theta}=\frac{\mu_{o} l_{p}}{2 \pi a}
$$

where $a$ is the distance between the center of the plasma and the coil. For example, for a plasma with a current of $1 \mathrm{MA}$ and a minor radius of $0.80 \mathrm{~m}$, the poloidal field intensity at the nearest coil from the plasma is approximately $0.25 \mathrm{~T}$.

Using the fast data, behaviour of the fluctuation level can be studied with a better time resolution. Fast Fourier Transforms (FFT's) may be used to obtain the frequency spectrum on any Mirnov coil. Then, the time evolution of the frequency spectrum during an impurity injection, a gas puff, or plasma motion can be investigated in detail.

Finally, in order to study the different models (temperature or pressure-driven fluctuations, change in scale length or change in mode location) knowledge of the electron temperature and the electron density 
profiles is required. Thomson scattering data were mostly used. However, for the study of time evolution of fluctuations, time-resolved temperature profile data from the electron cyclotron emission (ECE) radiometer were used. Similarly, the multichannel infrared interferometer (MIRI) gives time-resolved line-integrated density profiles.

IMPURITY INJECTION

The laser blow-off technique [2-4] is used on TFTR to inject elements of $Z$ number ranging from 21 to 34 (i.e., Sc,Fe,Ni,Cu,Ge, and $\mathrm{Se}$ ). A Q-switched nuby laser is used to vaporize a metallic film deposited on a glass slide, thus injecting metallic impurity into the plasma. The ionization time as exhibited by the peak on the signal of the bolometer is of the order of 15 to $20 \mathrm{~ms}$. The quantity of impurities injected varied and was evaluated by the rise in the bolometric total radiated power.

The interesting observation shown in figure 2 is that the magnetic fluctuation level decreases dramatically when the impurity is injected. However, as shown, plasma current, line-integrated density (through the center), major and minor radii (calculated geometricaliy from the major radius) do not change significantly during the injection (usually less than $1 \%$ ). The flucluation level recovers fully after different periods of time following closely the time history of the radiated power signal. A more detailed study of the impurities behaviour can be found in poierences 5 and 6.

The level of the bolometric power is taken to be a measure of the quantity of impurity injected. However, the exact relation between the amount of impurities and the rise in power radiated is unknown and subject to a lot of different factors. In the different figures. Prad represents the background total radiated power (i.e., before the injection) and $\Delta P_{\text {rad }}$, the rise over the background radiated power.

It was observed that a larger relative rise in the radiated power, (indicating more impurities injected) gave a larger drop in the fluctuation level. In figures 3 and 4 is plotted the variation of the relative drop of the fluctuation level versus the relative rise in the total power 
radiated. Two scales, linear (fig. 3) and square root (fig. 4) dependence, are used for the $x$ axis. The second gives a very good correlation. It is unclear whether or not the plasma current affects the scaling. The relative drop does not seem to be related to the mass of the element injected or to the type of plasme (i.e., $\mathrm{He}$ or D).

With data taken at a fast digitizing rate during the injection (at $100 \mathrm{kHz}$ ), the time evolution of the frequency spectrum can be studied. With an FFT done before and right after the injection, as shown in figure 5, a constant drop for all frequencies covered (1 to $50 \mathrm{kHz}$, the Nyquist cut-off frequency) is observed. The temperature profile peaks up significantly right after the injection. The plasma edge cools off relatively rapidly whereas the plasma center cools $20-30 \mathrm{~ms}$ later. This introduces some ciranges in the temperature gradient as a function of time. The edge electron density tends to remain about the same.

\section{GAS PUFFING}

By the use of a secondary gas injection valve on TFTR, small quantities of gas (usually less than 2 Torr-liters, nitrogen equivalent) can be intraduced into the vacuum vessel. In our case the gases injected were hydrogen $(H)$ and neon (Ne). Flow rate (Up to 16 Torr-liters/sec in $\mathrm{N}_{2}$ gauge equivalent, which differs from $\mathrm{H}$ and $\mathrm{Ne}$ flow rates by a factor of 4 and 6 less, respectively.) and puff duration are controlled and predetermined. Consequently, diflerent experiments can be performed by varying the total amount, the flow rate, or the puff duration. in TFTR experiments we used flow rates between 1 and 16 Torr-liters/sec and puff duration between 30 and $120 \mathrm{~ms}$.

A dramatic effect of a gas puff on the magnetic fluctuation level is shown in figure 6. Again, plasma current, major and minor radii do not change signilicantly (less than $1 \%$ ) during the event. The fluctuation level recovers in different times depending on the time history of the puffing and on the gas injected. The recycling capability of the walls [5] also introduces some differences in the behaviour compared to the impurity injection (by the laser blow-off technique). 
In order to evaluate the quantity of gas that effectively penetrated the plasma (some of the gas may actually stay in the scrape-off layer), the rise in the line density (through the center) was used as the measure of the gas input into the discharge. As for the previous case, plotted in figure 7 is the relative drop in the fluctuation level as a function of the rise in the electron line density. The variation is approximately linear, but it depends strongly on the type of gas introduced. The big difference between $\mathrm{H}$ and $\mathrm{Ne}$ originates mostly from their different number of electrons and ionization energies.

Further study of this phenomenon is possible by considering three consecutive shots where only the amount of gas injected varied (in this case neon). The drops in the magnelic fluctuations, as seen at the top of figure 8 are clearly different. However, as shown in the second plot of figure 8 , which indicates the flow rate as a function of time, it is unclear whether the flow rate or the total amount of gas is responsible for these drops. The use of the line-integrated density (at the bottom of ligure 8) clearly avoids that problem. The signal of the flow rate, which originates from the secondary valve, does not indicate accurately the amount of gas that actually penetrates into the plasma. It is also remarkable that an increase of $10 \%$ in the line density can produce a drop in the magnetic fluctuations of up to more than $90 \%$.

As in the laser blow-off case, the special case of neon puffing (as shown in figures 9 and 10) gives a similar square root dependence (with different magnitude) of the relative drop in the fluctuation level as a function of the relative rise in the power radiated. Therefore, this is evidence of a connection between the two experiments.

\section{FLUCTUATIONS RADIAL SCALE LENGTH}

An important clue for finding the radial location of the magnetic fluctuations is the scaling of the fluctuation amplitude with distance from the plasma. Because of the complexity of the tokamak geometry, only an approximation can be obtained. The simplified case of a 
straight fokamak (cylinder with axisymmetry, $k_{2}=0$ ) is here the appropriate approximation. In that geometry the plasma is located at the center of the cylinder and is surrounded by a vacuum. The vacuum magnetic field is known to be of the form of:

$$
\widetilde{B}_{\theta}(r)-A r^{m \cdot 1}+C r^{-(m+1)}
$$

where the origin is located at the center of the plasma. Outside the plasma the contribution will tome from the second term of the abcve expression. However, the number of coils (set of 15 coils. poloidally) is too low for an exact resolution of mode number which is believed to be greater than 15 (see bolow). Also, in a tokamak, in general, the toroidicity and the Shafranov shilt introduce new terms, making the expression for $\widetilde{B}_{\theta}(r)$ much more complex. However, here, Eq. $(3)$ is used as a firsi approximation.

This simple geometry allows us to use the full polnidal array of coils in order to estimate the scaliri. This estimaie does not consider the effects of a not perfectly conducting vall and of a nor.-zero toroidal wavenumber $\left(k_{2}\right)$. The $m$ number is then overestimated (at most by a factor of 2) as pointed out by $L$. Giannone[7]. As the coils are located at different distances from the center of the plasma, the absolute fluctuation level as a function of the distance of each coil to the center of the flux surfaces (plasma cenier) san be piotted. In figure 11 is plotted $\dot{B}$ versus the coil distance from the center of the piasma $\left(f_{\text {coil }}\right)$ before and right after an impurity injection. From that figure it is elear that the fluctuation level drops after the injection by a factor of more than two. The curve flattens at large distances, because of the noise level of the diagnostic. The scaiing number $(\sim m)$, obtained from the slope of the curve in figure 11, lies between 15 and 25 . We also observe that ine slope is not changing significantly with the injection of impurities, implying that the " $m$ " number remains the sanı.

With such a drastic radial scale length, we believe that the fluctuations pickeo up by the Mirriov coils are voming from a region very close to the edge. 
Three different models for interpreting the behaviour of the flucluation level during the different experiments are considered. The results obtained are used to evaluate the validity of each model.

The first model suggests that the inagnetic fluctuations are reiated somehow to the sdge electron temperature or to the electron pressure. The modes may be driven direcily by $T_{0}$ or $P_{\theta}$, or by their gradient $(\partial r)$ or by their logarithmic derivative $\left(\partial \ln / \partial_{r}\right)$. Any decrease in one of these factors would cause a consequential deciease in the magnetic fluctuation.

In the second model the variations could be explained by a change in the scale length as represented here by the " $m$ " number of the radial scale length. For examp!e, if $m$ increases significantly, the fluctuation level would decrease faster because $\widetilde{\mathrm{B}}_{\theta}(r)$ scales mainly as $\mathrm{Cr}-(m+1)$ [eq. (3)]. We assume that the other parameters remain constant [i.e., the constants $A$ and $C$ in eq. (3)].

The third model associates the fluctuation mode with a specilic region of the plasma. In that case. moving the edge of the plasma or inducing a similar motion by cooling effects can induce a change in the fluctuation level. This implies that the mode may move, i.e., move away from a coil. A shrinkage in the temperature profile or a plasma motion would be a good example of such movement.

\section{DISCUSSION}

A study of the correlation of the magnetic fluctuation level vith $P_{\theta}$ or $T_{\theta}$ " $r$ any of their derivatives) can be done on shots available on TFTR. However, special care must be taken for shots involving neutral-beam injection heating. Fluctuations picked up by the Mirnov coils may have different origins than in the ohmic-heated case. 
We would expect to obtain from the first model a relation between the fluctuation level and the electron temperature or pressure. W:th the high radial scale length obtained previously it is believed that fluctuations originate from a region very close to the plasma edge. In that region problems arise with the availability of electron temperature and density data. In orter to have a coherent set of data, the temperature and density were taken at a distance of $15 \mathrm{~cm}$ (compared to the minor radius which is usually $80 \mathrm{~cm}$ ) from the edge inside the plasma.

Usually a data point per shot (i.e., with Thomson scattering) is obtained; but in some cases, when the electron cyclotron, emission (ECE) measurements are available, a time evolution can be fellowed and is added directly to the database.

No correlation was found between $\widetilde{\mathrm{B}}_{\theta}$ and $\mathrm{T}_{\theta}$ at the edge. Although the temperature is also dropping with the fluctuations, no general scaling was found. However, as seen in figure 12 a good correlation was found with the electron pressure and is even better in ohmic discharges only (filled circles), including gas pulf and impurity injection shols.

The second model involves changes in the scale length. The magnetic fluctuation level was found to behave approximately as $C_{r}-(m+1)$ (the other factor being negligible). A decrease in the fluctuation level would then mean an increase in the $m$ number. Referring to figure 11 , a drop of 50\% in the signal is observed, for any coil well above the noise level. In order to obtain such a decrease $(50 \%), m$ must increase to approximately 100 trom between 15 and 25 . Figure 11 clearly does not show such a dramatic change in the slope of the fall-off curve. Therefore. the second model is rejected.

The third model was studied mainly with the use of free expansion experiments. In these experimients the plasma was moved slowly (The minor radius was increased from $0.67 \mathrm{~m}$ to $0.80 \mathrm{~m}$ ) so that the oufer flux surface (where we found the fluctuation modes to be located) stayed in permanent contact with the inner wall (a! the 0 degree location). 
Experimentally, it was observed that for many coils. especially those on the inboard side, which are closest to the plasma (i.e., $0,22.5,45,315$, and 337.5 degree coils as in figure 1), the magnetic fluctuation level was dropping (some coil signals remained constant but none increased). So, even though the plasma enge was coming cluser to some coils (i.e., 315 and 45 degree coils), the fluctuation level was decreasing. Observed also was a slight decrease in the signal of the coil located on the outer wall when the plasma edge was coming closer. Clearly the behaviour of the magnetic fluctuations cannot be explained by a possible motion of the mode location.

On the other hand, the free expansion experiment agrees with the first model. During the expansion the plasma pressure near the edge drops substantially in a way similar to the fluctuation level.

\section{CONCLUSION}

Experiments, such as imp:arity injection, gas puff, and free expansion, cause decreases in the magnetic fluctuation level. The eifest is often dramatic (drop of $\mathbf{5 0 \%}$ or more) and very sudden.

The amplitude of the drops were found to be strongly related to the quantity of gas or impurity injected into the plasma. The relative drop in the fluctuation level, in the case of impurity injection. was shown to be proportional to the square root of the rise in the radiated power, which is the best evaluation of the amount of impurity injecied. In the case of the gas pufis, the relative drop was proportional to the rise in the electron ine density.

For these events the radial scale length (" $m "$ number) and the frequency spectrum did not change significantly.

Finally, the magnetic fluctuations seen by a Mirnov coil may come from a region at or inside the plasma edge and are driven by the local pressure. 


\section{ACKNOWLEGMENTS}

The authe:s wculd like to expiess their gratitude towards the TFTR group. This work was alnported by iJS D.O.E. contract No DE-AC02-76-CHO-3073.

\section{REFERENCES}

[1] E. FREDRICKSON, Rev. Sci. Instrum. 57 (1986) 2084.

[2] E. S. MARMAR, Rev. Sci. Instrum. 46 (1975) 1149.

[3] S. COHEN et al. J. Vec. Sci. Technol. 20 (1982) 1226.

[4] D. MANOS and D. RUZIC, J. Vac. Sci. Technol. 20 (1982) 1230.

[5! E.S. MARMAR, J.E.AICE, J.L. TERRY and F.H. SEGUIN, Nudi. Fusion 22 (1982) 1567.

[6! B.C. STRATTCN, S.A. COHEN el al, J. Nucl. Matenals 145-147 (1987) 587.

[7] L.GIANNONE et al. 15th European Conference on Controlled Fusion and Plasma Physics, Dubrovnik vol.3 (1988) 1143. 


\section{FIGURE CAPTIONS}

figure 1. Set of 15 poloidal Mirnov coils locaied on the inside wall of TFTR. The coils can be as near as $10 \mathrm{~cm}$ to the edge of the plasma. They measure the magnetic fluctuations in the poloidal direction. The comb filter is usually connected to coil 1 ( 0 degree coil), located on the inner wall of TFTR.

figure 2. Plot of the different plasma parameters during a TFTR shot with an impurity injection. Selenium was injected at $4.2 \mathrm{sec}$. Electronic temperature is taken $a^{*} 10 \mathrm{~cm}$ from the center of the plasma.

figure 3. Plot of the relative drop in the fluctuation level as a function of the relative rise in the radiated power (linear dependence).

figure 4. Plot of the relative drop in the fluctuation level as a function of the relative rise in the radiated power (square root dependence). A least square regression shows a very good linear relation.

figure 5. FFT's (fast Fourier transform) performed on the raw signal before and after $(-15 \mathrm{~ms})$ the injection of $\mathrm{Ge}$ into the plasma.

figure 6. Plot of the different plasma parameters for a discharge with a gas puif. A total of 0.465 Torr-liters of hydrogen (nitrogen gauge equivalent) was injected at $4.2 \mathrm{sec}$ in a deuterium plasma. Electronic temperature is taken at $10 \mathrm{~cm}$ from the center of the plasma.

figure 7. Plot of the relative drop in the fluctuation level as a function of the increase in the line-integrated density.

figure 8. Comparison of neon injection in a deuterium plasina. Conditions are similar but three different amounts of gas were injected. Flow rates are in nitrogen gauge equivalent. 
figure 9. Scaling of the relative drop of the fluctuation level as a function of the relative rise in the radiated power for the special case of neon puffing (linear dependence).

figure 10. Scaling of the relative drop in the fluctuation level as a function of the relative rise in the radiated power for the special case of neon puffing (square root dependence).

figure 11. Plot of the magnetic fluctuations (full spectrum) taken from different poloidal locations on the torus wall. The coils are at different distances from the plasma center which vary from 91 to $131 \mathrm{~cm}$ for a plasma with a minor radius of $80 \mathrm{~cm}$. Titanium was injectad in this shot.

figure 12. Plot of the local pressure at $R \sim 1.80 \mathrm{~m}, 15 \mathrm{~cm}$ inside the plasma edgn, versus the magnetic fluctuations (at $100-150 \mathrm{kHz}$ ). 


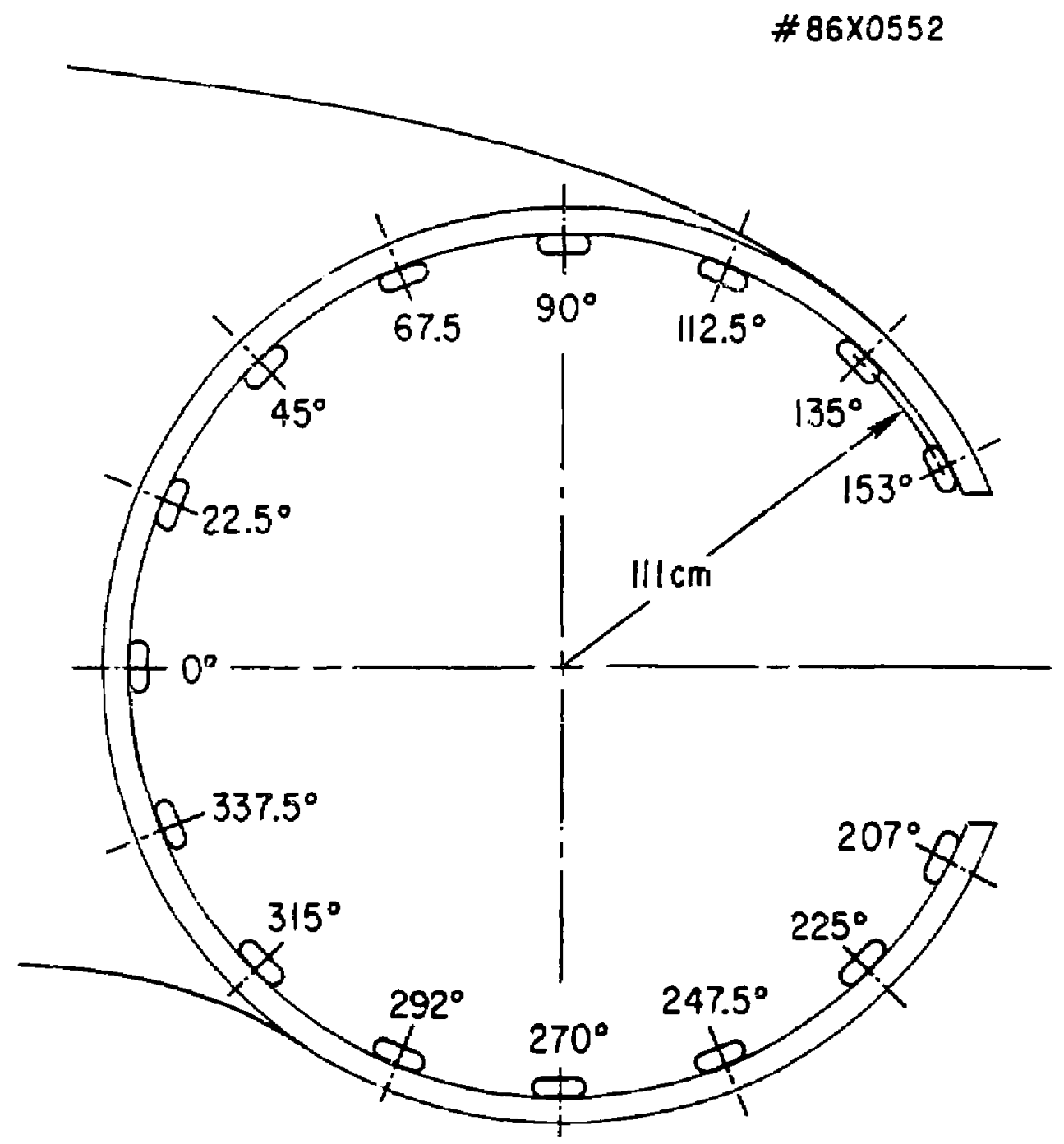

Eig. 1 


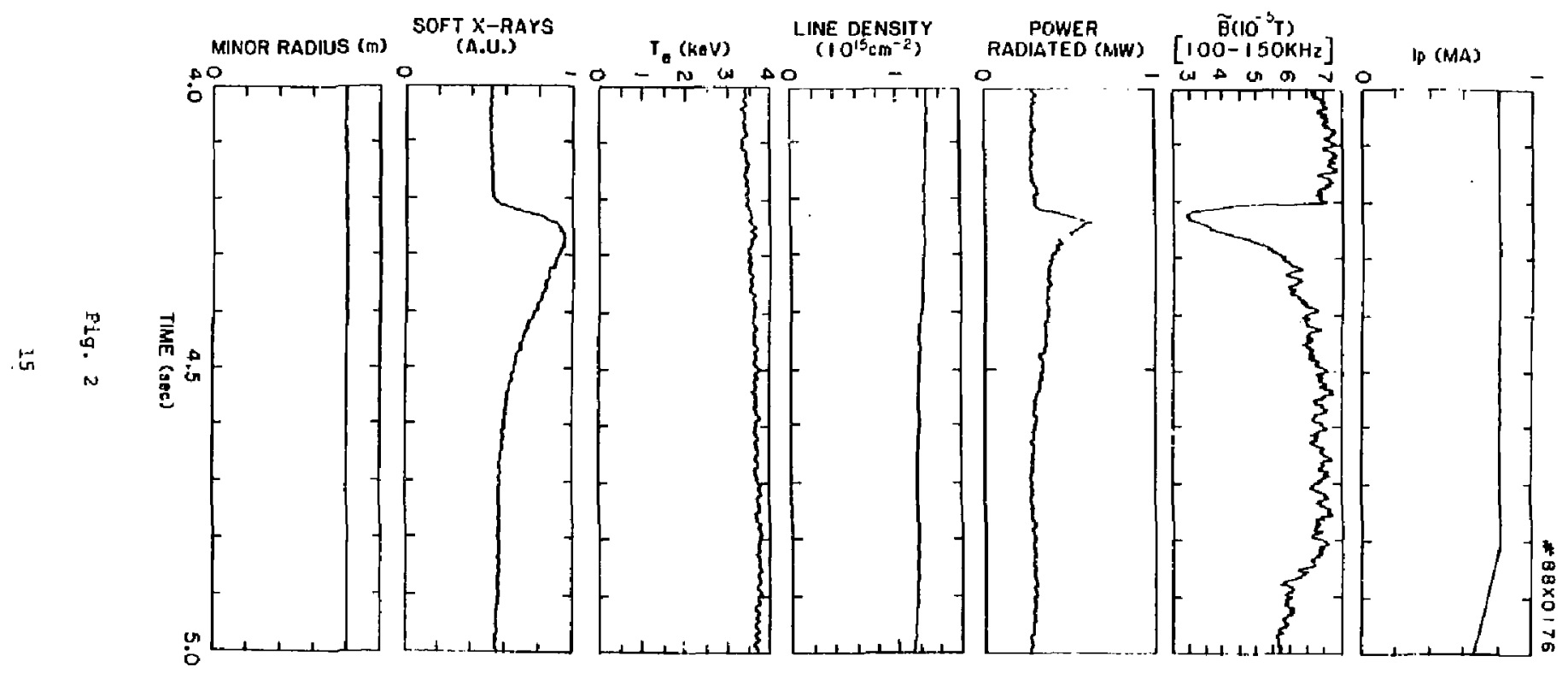




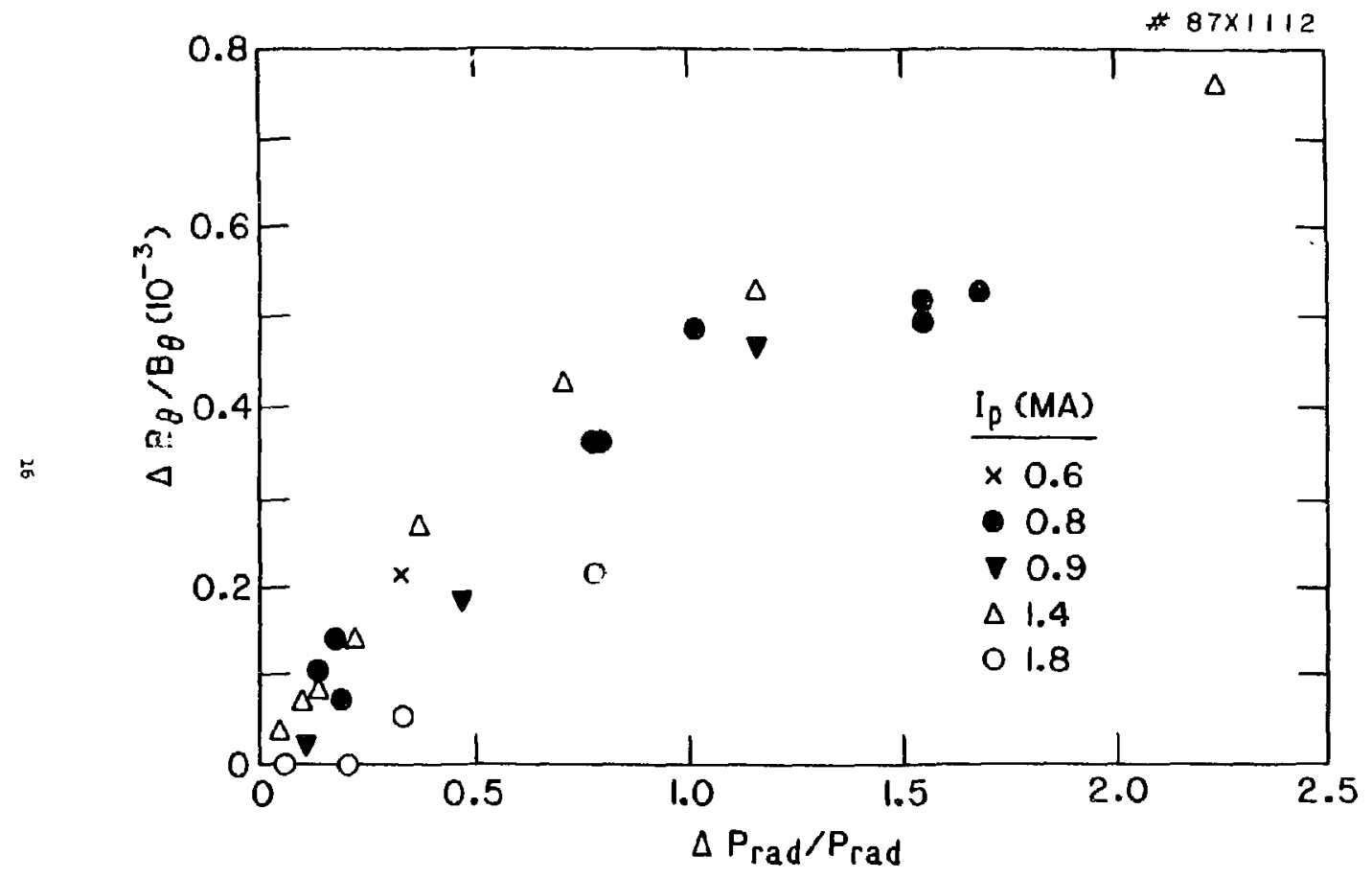

Fig. 3 


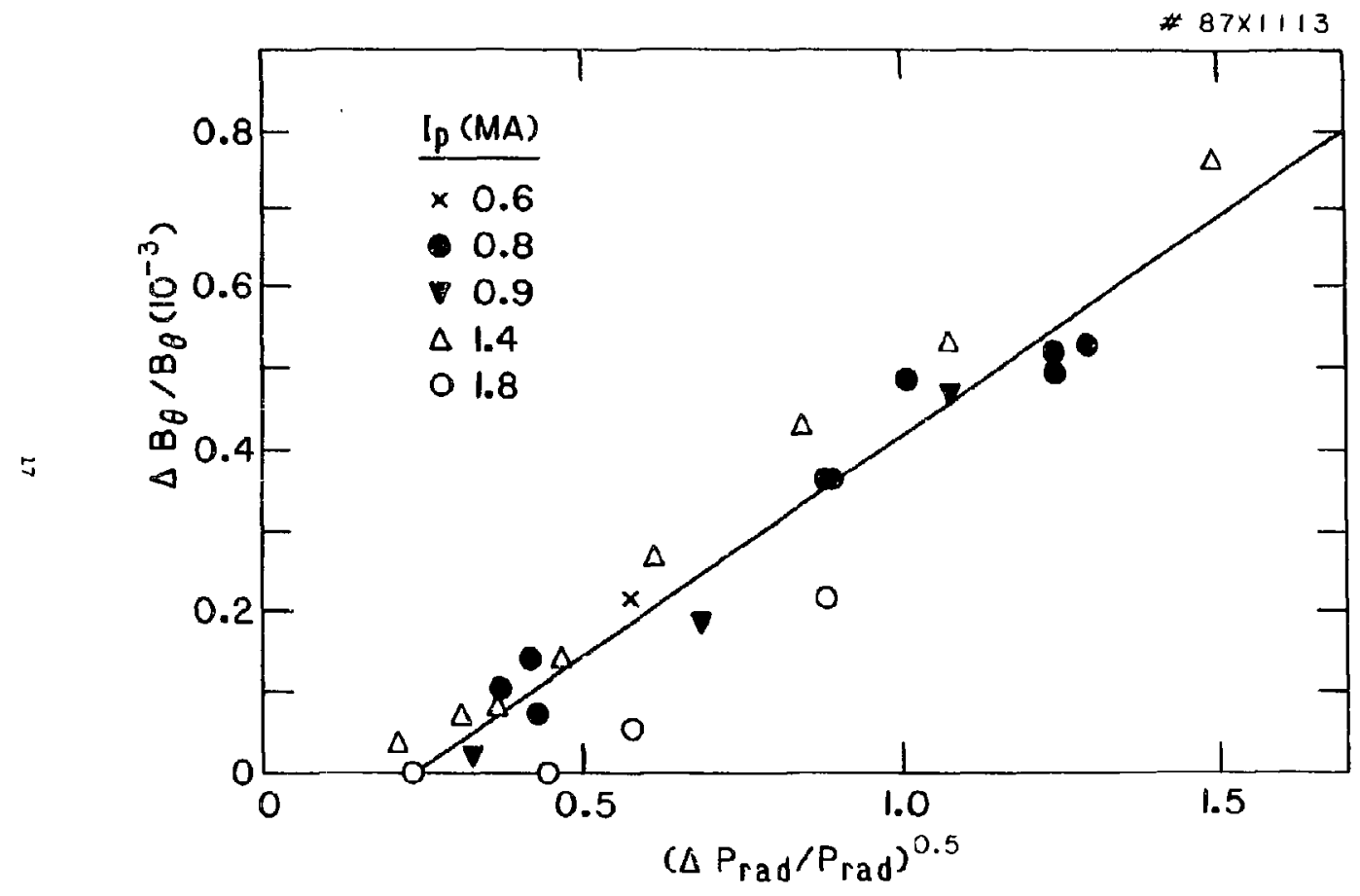




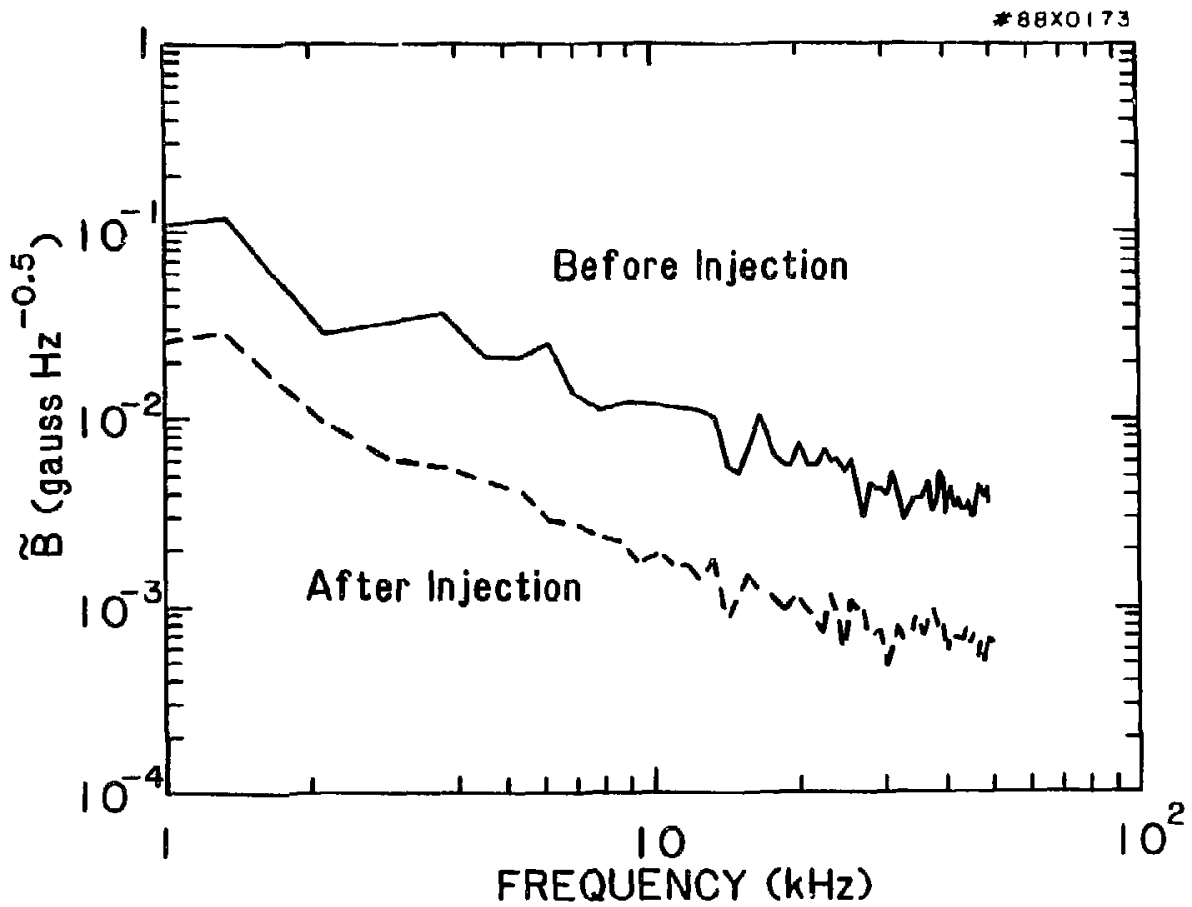

Fig. 5 


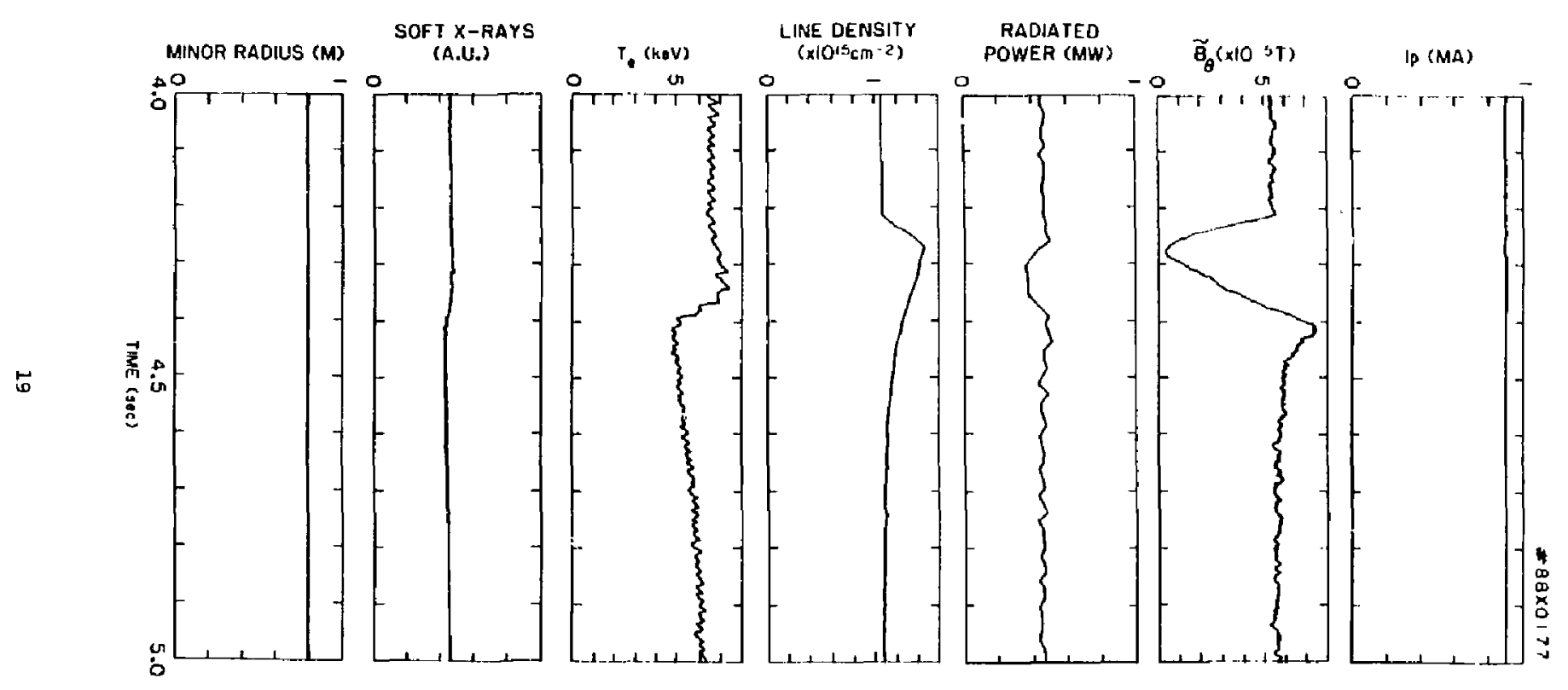




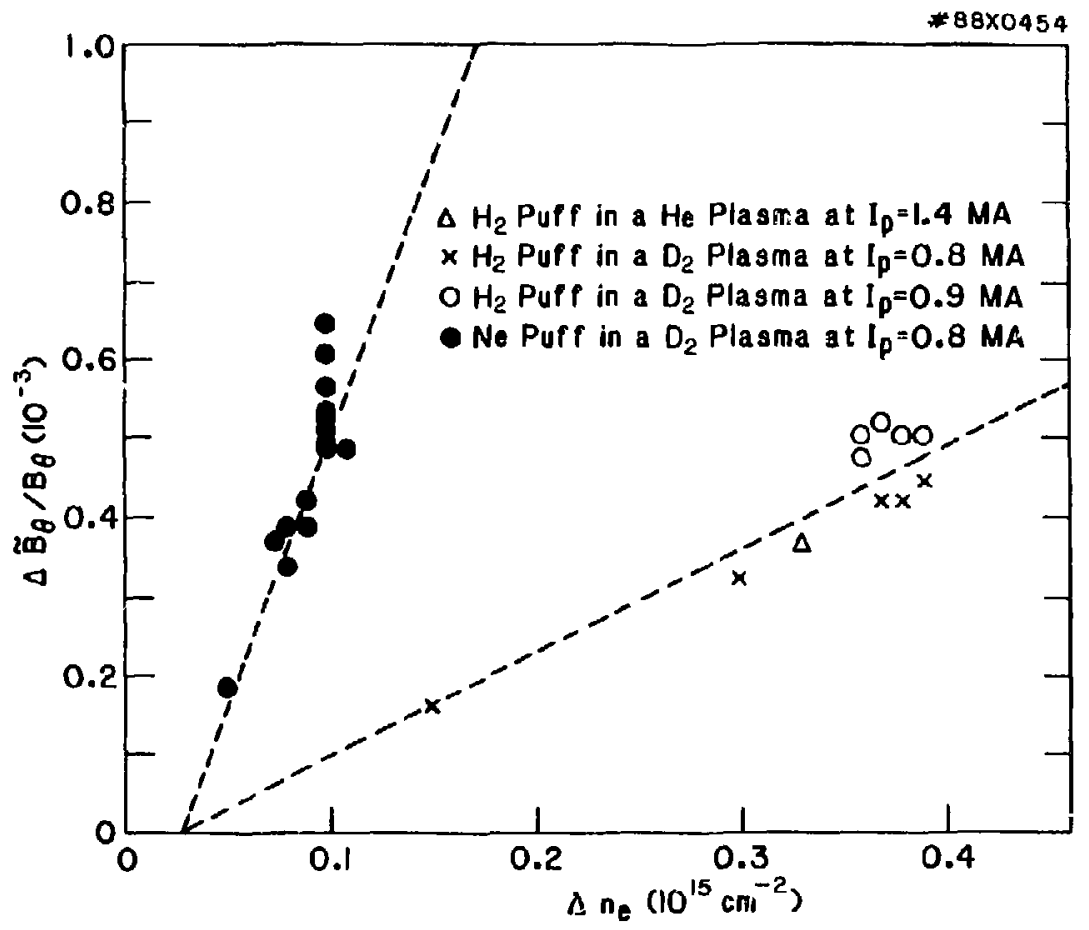

vic. 7 

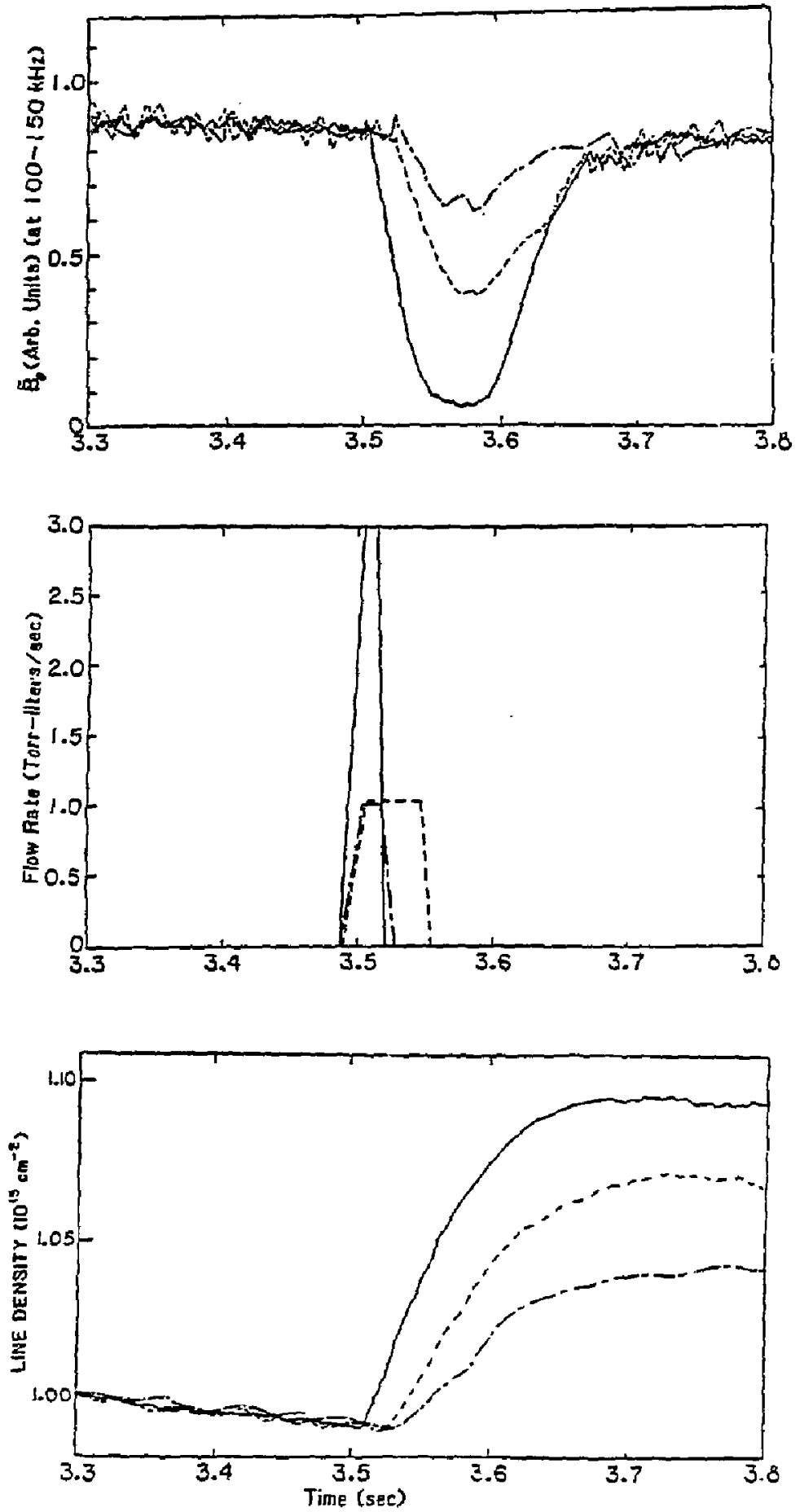


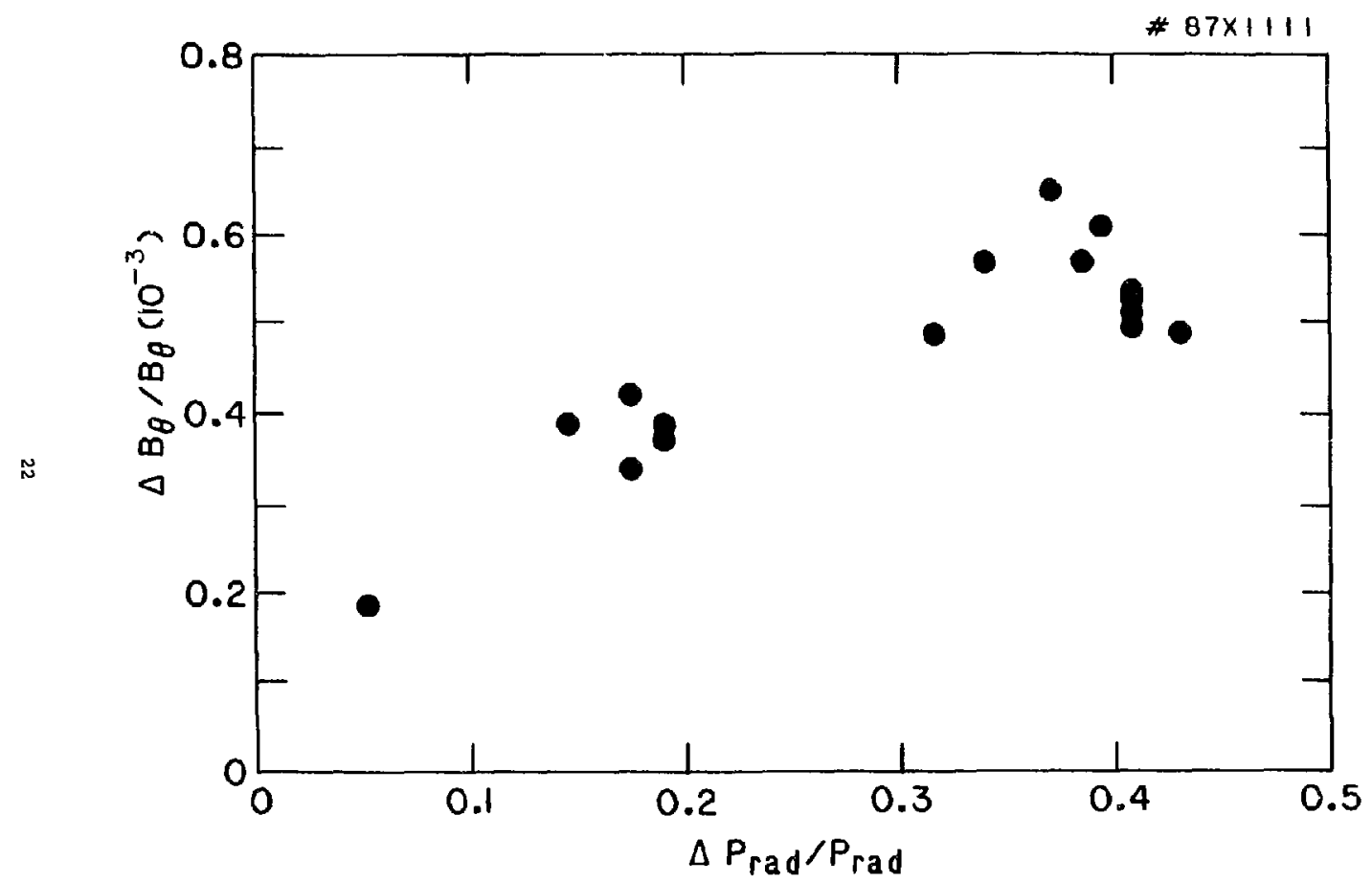

Fig. 9 


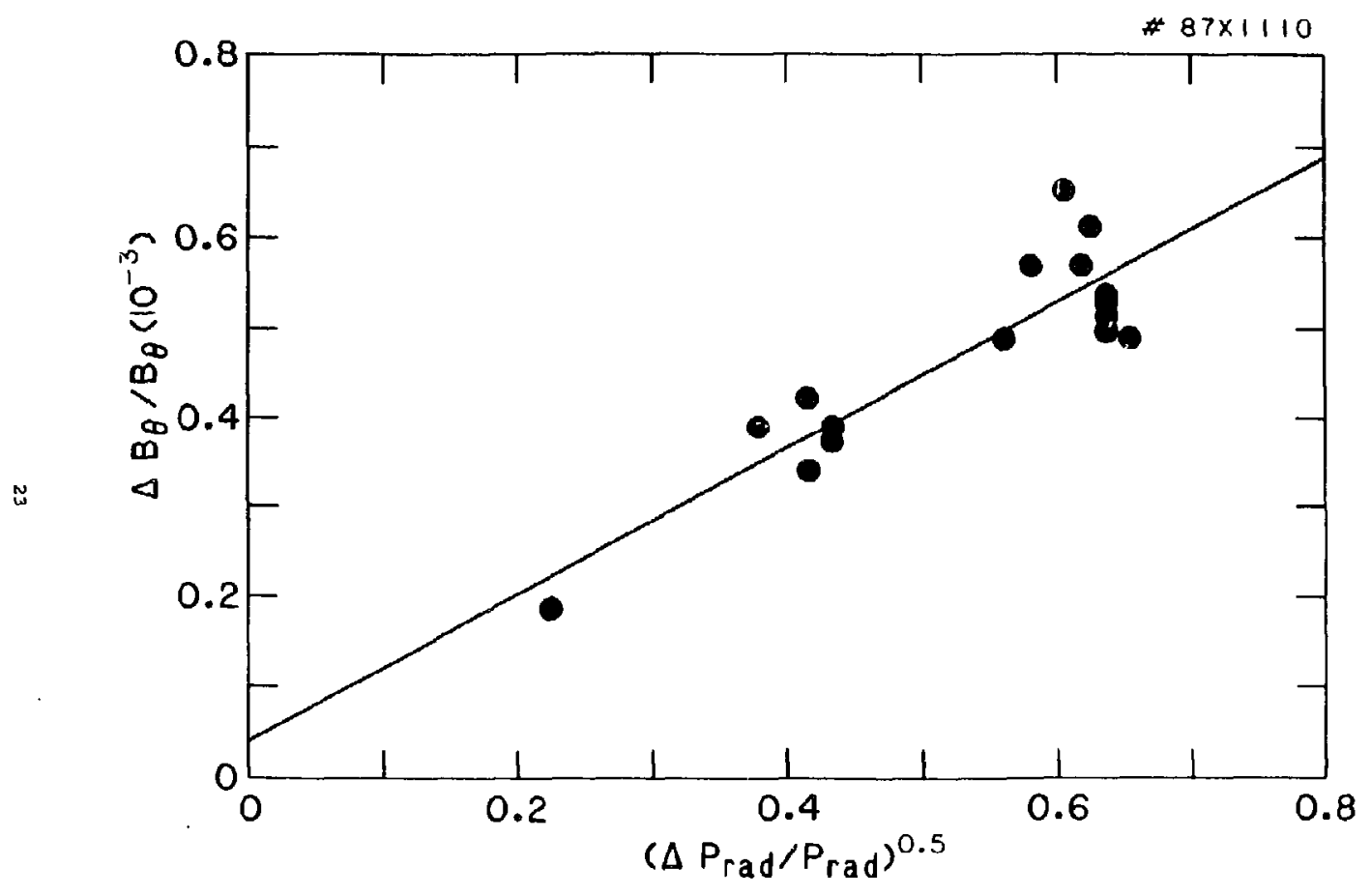




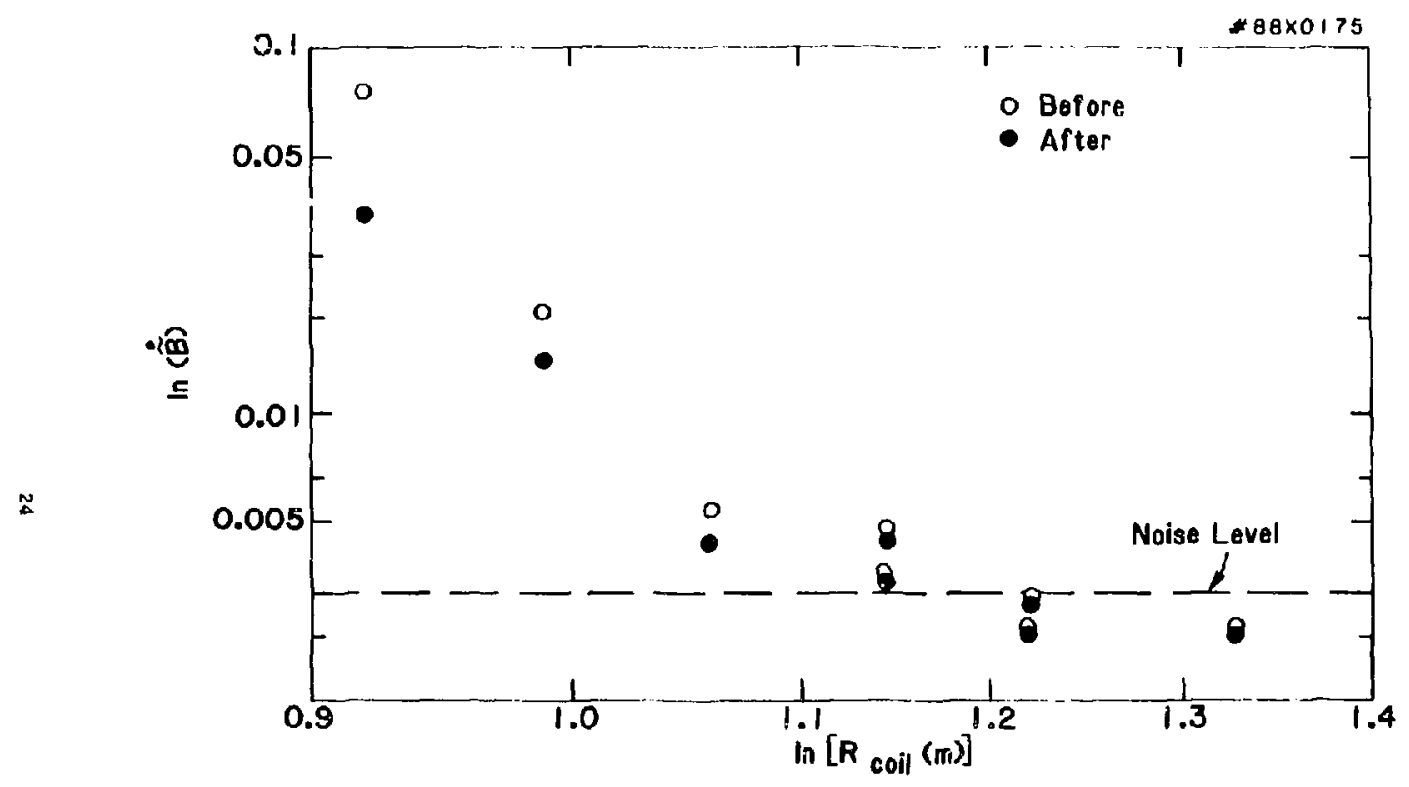

Fig. 11 


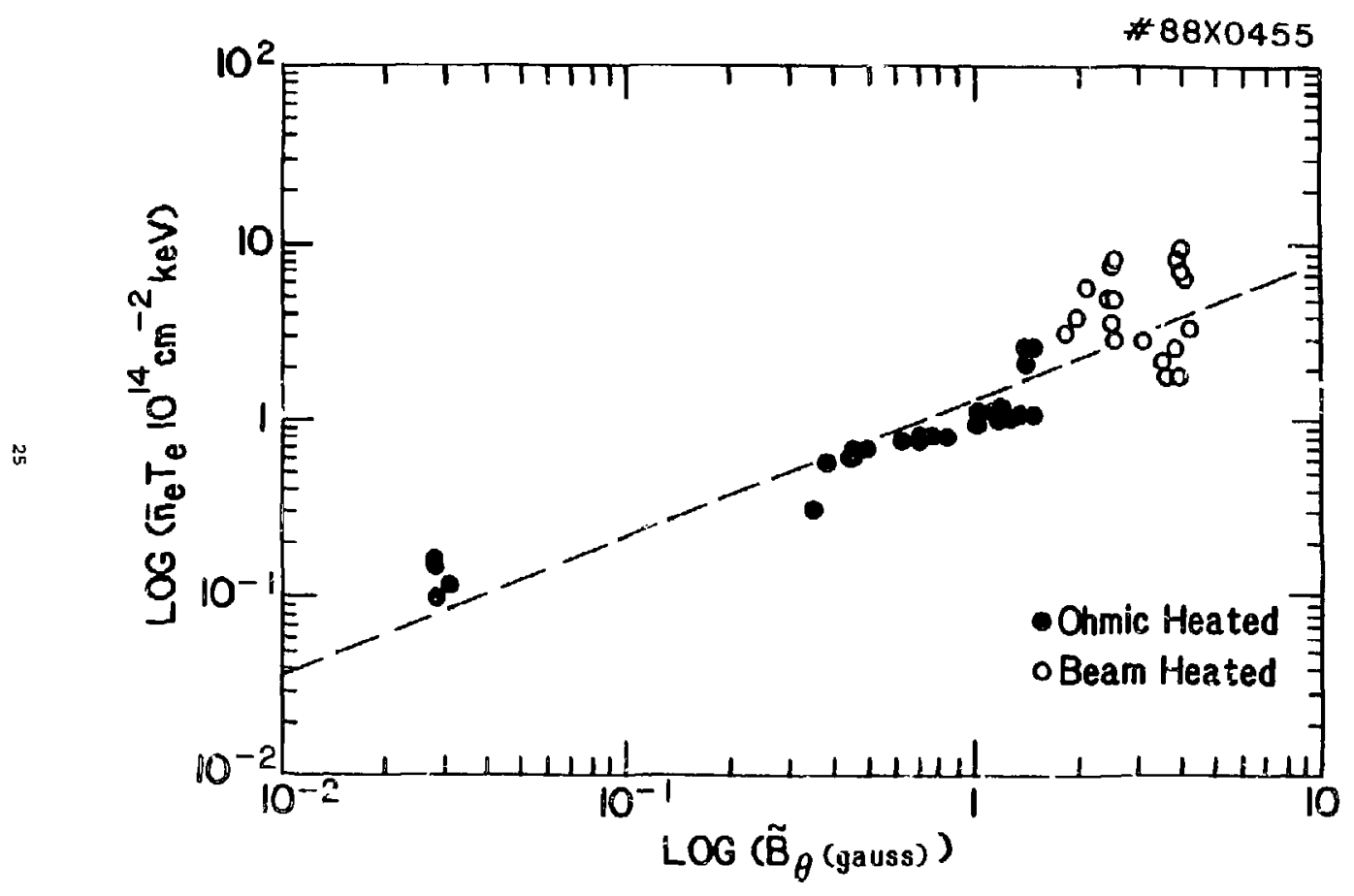

